coronary disease (4.78\%, p 0.867), hypertension (39.17\%, p 0.51), congestive heart failure $(0.71 \%$, p 0.987$)$, chronic renal failure $(0.49 \%$, p 0.596), tobacco use $(26.65 \%, \mathrm{p} 0.307)$ and number of consultations in the previous year (mean 0.53, SD 0.01, p 0.19). A total of 4237 visited their PCPs from 1 January 2010 to 26 August 2010. Patients cared for by clinicians randomised to the intervention group had significantly more mammograms performed $(18.8 \%$ vs $15.9 \% \mathrm{p}=0.047)$.

Conclusion We credit the decision support system with promoting completion of almost 30 delinquent mammograms per 1000 women who consulted their PCPs during an eight-month period.

\section{P1-165 DIFFERENCES IN MATERNITY SERVICE USE BETWEEN POLISH MIGRANTS AND THE HOST SCOTTISH POPULATION}

doi:10.1136/jech.2011.142976d.58

${ }^{1} \mathrm{D}$ Gorman, ${ }^{*}{ }^{1} \mathrm{~S} V$ Katikireddi, ${ }^{2} \mathrm{C}$ Morris, ${ }^{2} \mathrm{~J}$ Chalmers, ${ }^{1} \mathrm{~J}$ Sim, ${ }^{1} \mathrm{R}$ Hughes. ${ }^{1} \mathrm{NHS}$ Lothian, Edinburgh, UK; ${ }^{2}$ Information Services Division, NHS Services Scotland, Edinburgh, UK

Introduction Some migrant groups in Europe experience excess maternal morbidity and different processes may influence outcomes for specific groups. Polish women form the largest migrant group in Scotland with little data comparing their maternity experience with Scottish-born women. Qualitative findings suggest if women negotiated deliveries based on Polish not Scottish norms, they would have higher caesarean section and lower forceps deliveries rates than Scots. To assess this we compared data on access to care and maternity outcomes to identify any patterns associated with Polish migrants.

Methods NHS Scotland records detail about maternity care and maternal country of birth is required at birth registration. We matched these two datasets identifying mothers born in Poland and Scotland. Mode of delivery, use of analgesia, gestation, birth weight and proportion of live births were extracted for singleton deliveries and data adjusted for age, body mass index and smoking.

Results Polish migrants were younger, lighter, smoked less and booked maternity care later $(11.2 \%$ after 20 weeks vs $5.3 \%$ ). Caesarean section was less likely and forceps/ventouse more likely. Pregnancy outcomes tended to be better in Polish migrants with fewer low birth weight babies or neonatal admissions.

Conclusion Reduced Caesarian section rate and favourable birth and maternal outcomes in the Polish population, may be due to the 'healthy migrant' effect (as we were unable to adjust for education/ socioeconomic status) and residual confounding. Reasons for excess instrumental deliveries remain unclear. Later maternity booking reflects patterns in other migrant groups, and reinforces the importance of improving health system understanding.

\section{P1-166 EXPLAINING SOCIAL PATTERNING OF MORTALITY: THE ROLE OF LIFESTYLE}

doi:10.1136/jech.2011.142976d.59

${ }^{1} \mathrm{~L}$ Gray, ${ }^{*}{ }^{2} \mathrm{~J}$ Armstrong, ${ }^{2} \mathrm{Y}$ Brogan, ${ }^{3} \mathrm{~A}$ Sherriff, ${ }^{4} \mathrm{C}$ Bromley, ${ }^{1} \mathrm{~A}$ Leyland. ${ }^{1} \mathrm{M} R \mathrm{C} / \mathrm{CSO}$ Social and Public Health Sciences Unit, Glasgow, UK; ${ }^{2}$ School of Life Sciences, Glasgow Caledonian University, Glasgow, UK; ${ }^{3}$ Department of Dentistry and Medicine, University of Glasgow, Glasgow, UK; ${ }^{4}$ Scottish Centre for Social Research, Edinburgh, UK

Background Mortality is strongly correlated with socio-economic status (SES). Lifestyle factors impact on health and are also independently linked with SES, and thus may drive the mortality-SES associations. However, their relative individual and combined contributions to such inequalities have not been well quantified. We addressed this using nationally representative prospective data.

Methods Analyses were based on 6060 participants aged over 16 years in the 2003 Scottish Health Survey (60\% response) providing data on cigarette smoking status, weekly alcohol intake, physical activity levels, diet (quality index) and body mass index (BMI; weight/[height $]^{2}$ ), and consenting to linkage with mortality records (until 2008). Cox proportional hazards regression assessed the relative index of inequality (RII) in mortality by SES (occupational social class) and attenuation by lifestyle factors.

Results Mortality-SES patterns were clear (292 deaths; $p<0.001$ ) and did not differ by sex $(p=0.212)$. The inequality across the social classes was more than twofold [age-adjusted RII $\mathrm{HR}=2.26$; $95 \% \mathrm{CI}$ 1.47 to 3.47]. Mutually adjusting for lifestyle factors reduced differences by $49 \%$ [1.65; 1.06 to 2.57$]$, with the greatest individual impacts made by cigarette smoking (29\%), diet (27\%) and physical activity (20\%); alcohol consumption and BMI had lesser effect.

Conclusions Around half of the SES patterning of mortality was explained by these lifestyle factors. Our findings provide valuable insight on the relative impact of individual lifestyle factors-essential for tackling socio-economic inequalities in health-highlighting the importance of promoting healthy eating and physical activity as well as further reducing smoking.

\section{P1-167 INDIVIDUAL AND TERRITORIAL DISPARITIES IN THE KNOWLEDGE AND PRACTICES OF THE FRENCH NATIONAL NUTRITIONAL GUIDELINE « FRUITS AND VEGETABLES: AT LEAST FIVE EVERY DAY » IN PARIS METROPOLITAN AREA}

doi:10.1136/jech.2011.142976d.60

${ }^{1,2}{ }^{2}$ Grillo, ${ }^{1,2} \mathrm{~J}$ Martin, ${ }^{3} \mathrm{~A}$ Lhuissier, ${ }^{1,2} \mathrm{M}$ G Siriwardana, ${ }^{1,4} \mathrm{P}$ Chauvin.* ${ }^{1}$ INSERM U707, UMR-S 707, Research Team on the Social Determinants of Health and Healthcare, Paris, France; ${ }^{2}$ UPMC Université Paris 06, Faculté de Médicine Saint Antoine, Paris, France; ${ }^{3}$ INRA, Unité ALISS UR 1303, Ivry-sur-Seine, France; ${ }^{4}$ Hôpital Saint Antoine, Unité de santé publique, Paris, France

Introduction The ageing of the European population raises questions concerning the growing incidence of chronic diseases. Improving population health status, by acting on one of its most important determinants, that is, nutrition, was the target of the PNNS (Nutrition Health National Program) launched in 2001 in France. This study aimed to examine the individual and territorial characteristics associated with the PNNS guideline to eat: " at least five fruits and vegetables every day " (R5FV).

Methods The third wave of the SIRS cohort interviewed a representative sample of the general population of Paris metropolitan area (3084 adults) in 2009-2010 about its knowledge of the R5VF. Adherence to this guideline, and reasons for non-adherence, were questioned too. Regression models analysed factors associated with non-knowledge of the guideline.

Results The vast majority of the population (98\%) was familiar with the R5VF but only $50 \%$ adhered to it. Men ( $\mathrm{aOR}=2.58$ ), foreigners $(\mathrm{aOR}=5.53)$, people who had never worked $(\mathrm{aOR}=6.66)$, those who had only primary level education $(\mathrm{aOR}=3.47)$, those residing in underprivileged neighbourhoods $(\mathrm{aOR}=2.05)$ were more likely to ignore it. Among those who knew it, reasons declared for not adhering were: too complicated to manage (28\%), too expensive (26\%) and not desiring (17\%)

Conclusion This study outlined individual and territorial inequalities in the reception of PNNS nutritional messages related to consumption of $5 \mathrm{~F} \& \mathrm{~V}$ a day. Consideration of individuals' demographic, socioeconomic and residence characteristics can identify population groups that necessitate to be targeted in order that current nutritional know-how reaches all people, especially the disadvantaged. 